\title{
Discontinuities in the level density of small quantum dots under strong magnetic fields
}

\author{
Augusto Gonzalez* \\ Instituto de Cibernetica, Matematica y Fisica, Calle E 309, Vedado, Ciudad Habana, Cuba \\ Roberto Capote \\ Departamento de Física Atómica, Molecular y Nuclear, Universidad de Sevilla, Facultad de Física, AP 1065, E-41080 Sevilla, Spain
}

(Received 30 May 2002; published 30 September 2002)

\begin{abstract}
Exact diagonalization studies of the level density in a six-electron quantum dot under magnetic fields around $7 \mathrm{~T}$ ("filling factor" around $\frac{1}{2}$ ) are reported. In any spin-polarization channel, two regimes are visible in the dot excitation spectrum: one corresponding to interacting quasiparticles (i.e., composite fermions) for excitation energies below $0.4 \mathrm{meV}$, and a second one for energies above $0.4 \mathrm{meV}$, in which the level density (exponentially) increases at the same rate as in the noninteracting composite-fermion model.
\end{abstract}

DOI: 10.1103/PhysRevB.66.113311

PACS number(s): 73.21.La, 73.43.Lp, 73.63.Kv

The lowest-energy states of relatively small quantum dots (number of electrons $N_{e} \geqslant 3$ ) in strong magnetic fields have been qualitatively described whithin the composite-fermion picture. ${ }^{1-3}$ The position of cusps in the ground-state energy as a function of the angular momentum, and the spin quantum numbers of the low-lying excited states are nicely reproduced by this theory. The residual interaction between composite fermions (CF's) is expected to be a weak, contact interaction.

The present paper is aimed at giving a quantitative characterization of the density of energy levels of small quantum dots in an energy interval below $1-1.5 \mathrm{meV}$, where dozens or hundreds of states exist. Fully converged exact diagonalization results for a six-electron GaAs dot in magnetic fields corresponding to "filling factors" $\nu \approx \frac{1}{2}$ are presented below. The error in computing energy eigenvalues is estimated to be lower than $0.02 \mathrm{meV}$ for levels with excitation energies below $1 \mathrm{meV}$. The studied excitation energy range is small as compared to the cyclotronic $\left(\hbar \omega_{c}=12 \mathrm{meV}\right)$, Coulomb (8.4 $\mathrm{meV})$, or confinement $\left(\hbar \omega_{0}=3 \mathrm{meV}\right)$ energies of the model dot. Three Landau levels (LL's) are included in the calculations, and a $25-\mathrm{meV}$ cutoff in the energy of the noninteracting many-electron states used as basis functions ${ }^{4}$ allows us to deal with Hamiltonian matrices of dimension less than 850000 , which are diagonalized by means of a Lanczos algorithm. The main result of the paper is the existence of two regimes in the excitation spectra, corresponding to low $(\Delta E<0.4 \mathrm{meV})$ and intermediate $(\Delta E>0.4 \mathrm{meV})$ excitation energies, in which the level density increases at different rates. Thus, at an energy interval $\delta E$ around $0.4 \mathrm{meV}$ the level density experiences a "discontinuity."

The model parameters are similar to those used in Ref. 5. The confinement potential is parabolic. The bare Coulomb interaction is weakened by a factor 0.8 to approximately account for quasibidimensionality (instead of exact bidimensionality). The Zeeman energy is written in the form $0.01432 B s_{z} \mathrm{meV}$ ( $B$ in Tesla and $s_{z}= \pm \frac{1}{2}$ ), corresponding to a 8 -nm-width well in magnetic fields around $7 \mathrm{~T}^{6}$

The lowest-energy states in each angular momentum and spin-polarization tower (the yrast spectrum) for magnetic fields $B=7$ and $8 \mathrm{~T}$ are shown in Fig. 1. Energy jumps between adjacent angular momentum states are about 0.6
$\mathrm{meV}$ in the spin-polarized case, but roughly three times smaller in any other spin-polarization sector. At this point it is important to stress the role of the higher LL's in the energy eigenvalues. The absolute contribution of the second and third LL's is around $-0.4 \mathrm{meV}$, a magnitude much greater than the Zeeman splitting $(0.1 \mathrm{meV})$, and than the characteristic energy spacing between states near the absolute minimum. Excitation energies are pushed down $0.1-0.3 \mathrm{meV}$ by the higher LL's.

We show in Fig. 2 the number of states as a function of the excitation energy, $\Delta E$. In this figure, the reference energy

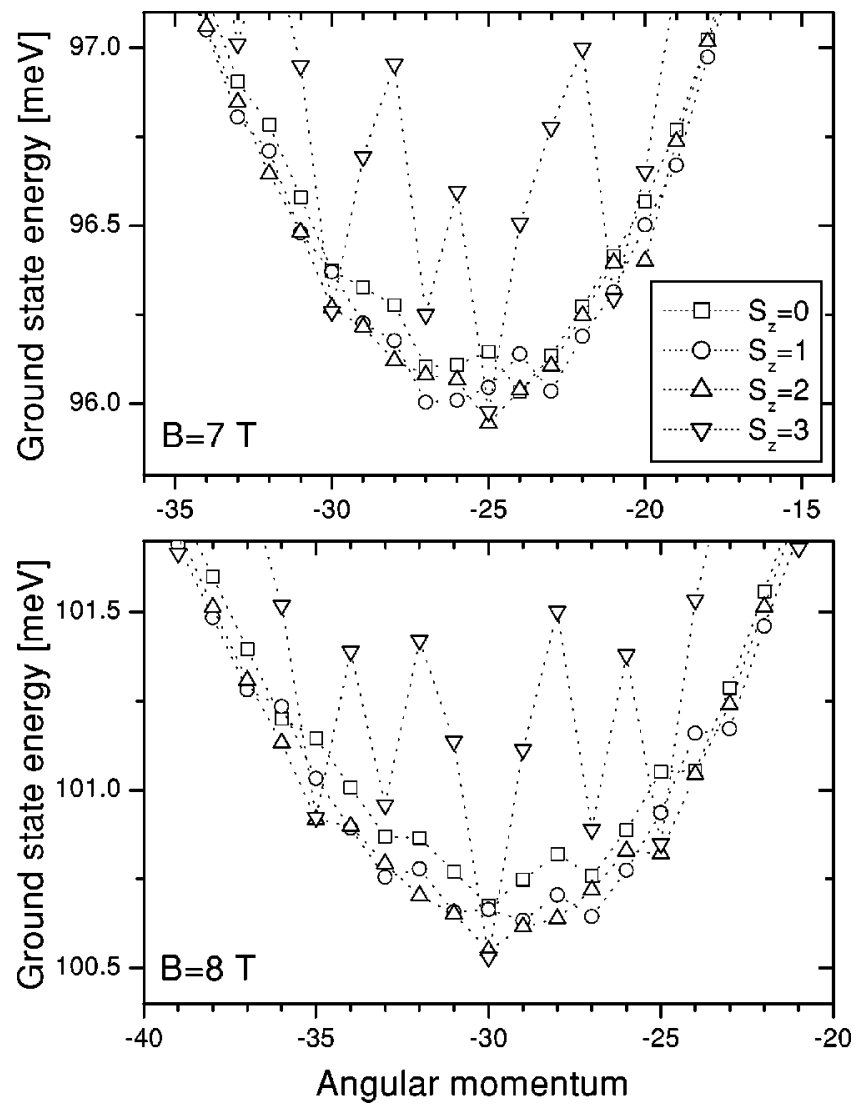

FIG. 1. The lowest-energy levels in each angular momentum and spin-polarization sectors. Lines are guides to the eyes. 

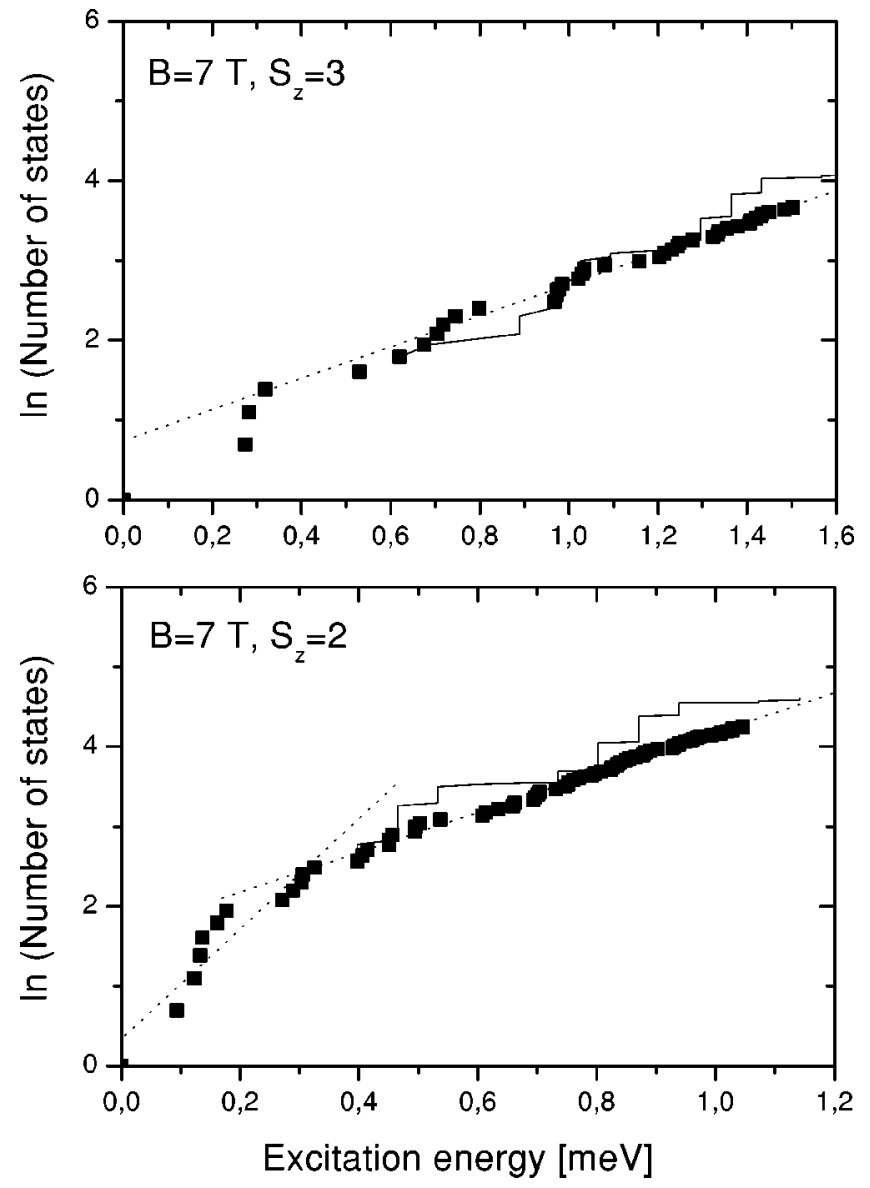

FIG. 2. The logarithm of the number of levels as a function of the excitation energy at $B=7 \mathrm{~T}$ : exact results (squares), constant temperature fits (dotted lines), and the shifted NICF curves (solid lines).

is the minimal-energy state within each polarization sector. At low and intermediate excitation energies the curves are well fitted by exponential functions, ${ }^{5}$

$$
n(\Delta E)=n_{0} \exp \frac{\Delta E}{\Theta}
$$

i.e., described by the so-called "constant-temperature approximation." "In the polarized case, there are only a few states for low excitation energies, thus we do not fit them. The fitting parameters $n_{0}$ and $\Theta$ are given in Table I. Primed quantities correspond to the low-energy sectors. Notice that the temperature parameter, $\Theta$, changes very little on going from $S_{z}=2$ to $S_{z}=0$ for a given $B$. Notice also the apparent jump of the temperature parameter at $\delta E$, which means a discontinuity in the level density.

The noninteracting CF (NICF) understanding of the excitation spectrum starts from a simplified first LL picture in which the excitation energy is written in the form ${ }^{2}$

$$
\Delta E=\hbar \omega_{C F} \Delta n_{L L}+\hbar \omega_{0}^{2} / \omega_{c} \Delta L
$$

$\Delta n_{L L}$ is the variation, with respect to the quasiparticle ground state, of the effective LL occupation numbers. By definition,
TABLE I. The level-density parameters $n_{0}$ and $\Theta$. Primed quantities correspond to excitation energies below $\delta E$.

\begin{tabular}{cccc}
\hline \hline$S_{z}$ & & $B=7 \mathrm{~T}$ & $B=8 \mathrm{~T}$ \\
\hline \multirow{3}{*}{3} & $\delta E$ & 0.30 & 0.45 \\
& $n_{0}$ & 2.099 & 1.712 \\
$\Theta$ & 0.510 & 0.443 \\
\hline \multirow{2}{*}{2} & $n_{0}^{\prime}$ & 1.398 & 1.614 \\
& $\Theta^{\prime}$ & 0.145 & 0.145 \\
& $\delta E$ & 0.35 & 0.30 \\
& $n_{0}$ & 5.341 & 5.241 \\
& $\Theta$ & 0.400 & 0.363 \\
\hline \multirow{3}{*}{1} & $n_{0}^{\prime}$ & 1.982 & 2.069 \\
& $\Theta^{\prime}$ & 0.107 & 0.085 \\
& $\delta E$ & 0.30 & 0.20 \\
& $n_{0}$ & 11.57 & 10.99 \\
& $\Theta$ & 0.398 & 0.344 \\
\hline \hline & $n_{0}^{\prime}$ & 1.506 & 1.635 \\
& $\Theta^{\prime}$ & 0.113 & 0.100 \\
& $\delta E$ & 0.40 & 0.25 \\
& $n_{0}$ & 14.74 & 12.90 \\
& $\Theta$ & 0.423 & 0.358 \\
\hline \hline
\end{tabular}

$\Delta L=|L|-\left|L_{g s}\right|$, where $L_{g s}$ is the ground-state angular momentum. $\hbar \omega_{c}$ is the electron cyclotronic energy in GaAs, and the effective cyclotronic energy of CF's is extracted from the polarized yrast spectrum, $S_{z}=3$, as ${ }^{2}$

$$
\hbar \omega_{C F}=E\left(L_{3}+1\right)+E\left(L_{3}-1\right)-2 E\left(L_{3}\right),
$$

where $L_{3}$ is the angular momentum of the lowest polarized state. It gives $\hbar \omega_{C F}=1.15$ and $1.19 \mathrm{meV}$ at $B=7$ and $8 \mathrm{~T}$, respectively. The quasiparticles are supposed to occupy states in effective Landau levels, which are separated by $\hbar \omega_{C F}$. The angular momentum of the quasiparticle system is $L_{C F}$ which, due to the special form of the variational CF wave function, is related to $L$ by $^{2}$

$$
L=-N_{e}\left(N_{e}-1\right)+L_{C F} .
$$

The NICF model gives qualitatively correct answers to questions such as the position of cusps in the yrast spectrum, the nature of the first excited states, etc. One may ask for other visible manifestations of the NICF in the spectrum, and indeed, it gives the correct $\Theta$ parameter at intermediate excitation energies, $\Delta E>\delta E$. In other words, the NICF curve growths exponentially with the same $\Theta$, although it is shifted with respect to the actual spectrum. This fact is illustrated in Fig. 2 also, where the NICF curve at a certain level (the sixth in the upper figure, for example) is forced to meet the actual curve. From this point on the two spectra have the same slope on average.

There is a natural interpretation of this behavior. In the ground and first excited states (low $\Delta E$ ), the quasiparticles form compact clusters ${ }^{2}$ and the interaction between CF's plays an important role. The low-energy parameter $\Theta^{\prime}$ is a 


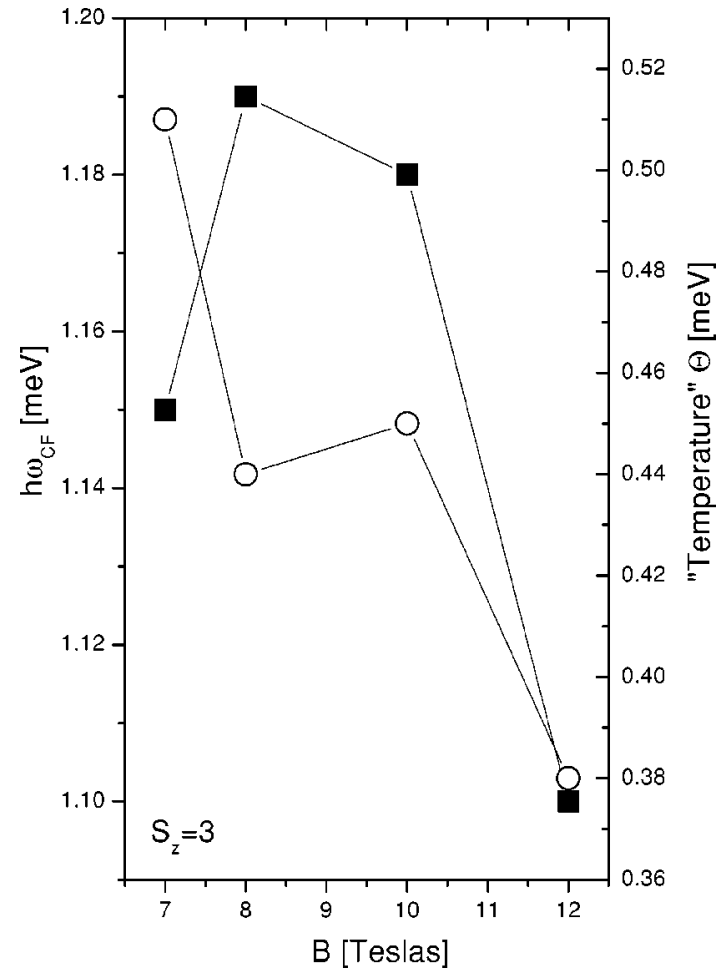

FIG. 3. The magnitudes $\hbar \omega_{C F}$ (squares) and $\Theta_{S_{z}=3}$ (circles) as a function of magnetic-field intensity $B$.

consequence of this interaction. On the other hand, at intermediate energies and assuming that the interaction is weak, one expects the level density to be dominated by the relevant energy scales, i.e., $\hbar \omega_{C F}$ and $\hbar \omega_{0}^{2} / \omega_{c}$. One may visualize this regime in terms of Rydberg-like excitations, in which a few noninteracting CF's orbit around a core of weakly interacting CF's. Energy differences will, of course, follow the NICF rules.

The conclusion to be extracted from this figure is that traces of the effective LL structure of CF's may also be looked for at intermediate excitation energies, $\Delta E$ $>0.4 \mathrm{meV}$. For $\Delta E<0.4 \mathrm{meV}$ the transition to a regime of weakly interacting quasiparticles takes place. Temperature parameters are different from both sides of $\delta E$, thus a discontinuity in the level density is expected.

The level density may be directly measured in Ramanscattering experiments under extreme resonance, where the Raman amplitude depends on the density of energy levels in final states. ${ }^{8}$ Magnetoconductance measurements under equilibrium ${ }^{9}$ or nonequilibrium conditions ${ }^{10}$ could also give evidence about the level-density behavior at intermediate excitation energies.

Finally, we show in Fig. 3 the magnitudes $\hbar \omega_{C F}$ and $\Theta_{S_{z}=3}$ in a wider magnetic-field intensity range, $7 \leqslant B$ $\leqslant 12$ T. Near $B=12 \mathrm{~T}$, where the "filling factor" is around $\frac{1}{3}$, there is a decrease of $\hbar \omega_{C F}$ which corresponds to an abrupt increase of the quasiparticle mass. ${ }^{11}$ A similar behavior is observed in $\Theta$, showing that the level-density parameter $\Theta$ depends on a negative power of $m_{C F}$.

Part of this work was carried out at the Abdus Salam ICTP. A. G. acknowledges the ICTP Associate and Federation Schemes for support. R. C. acknowledges support from the Ministerio de Educación, Deportes y Cultura de España, Secretaría de Estado de Educación y Universidades.
*Electronic address: agonzale@ @idet.icmf.inf.cu

†Permanent address: Centro de Estudios Aplicados al Desarrollo Nuclear, AP 100, Ciudad Habana, Cuba. Electronic address: rcapote@us.es

${ }^{1}$ C.W.J. Beenaker and B. Rejaei, Physica B 189, 147 (1993).

${ }^{2}$ J.K. Jain and T. Kawamura, Europhys. Lett. 29, 321 (1995); R.K. Kamilla and J.K. Jain, Phys. Rev. B 52, 2798 (1995).

${ }^{3}$ J.K. Jain and R.K. Kamilla, in Composite Fermions, edited by O. Heinonen (World Scientific, Singapore, 1988).

${ }^{4}$ The basis functions in a disk geometry are described in $\mathrm{T}$. Chakraborty and P. Pietilainen, in The Quantum Hall Effects (Springer, New York, 1996).
${ }^{5}$ A. Gonzalez and R. Capote, Physica E (Amsterdam) 10, 528 (2001).

${ }^{6}$ We use a parametrization by B. Rodriguez of the experimental $g$ values for quantum wells given in S.P. Najda et al., Phys. Rev. B 40, 6189 (1989); M.J. Snelling et al., ibid. 44, 11345 (1991); 45, 3922 (1992); N.J. Traynor et al., ibid. 55, 15701 (1997); M. Seck et al., ibid. 56, 7422 (1997).

${ }^{7}$ T. Ericson, Adv. Phys. 9, 425 (1960); A. Gilbert and A.G.W. Cameron, Can. J. Phys. 43, 1446 (1965).

${ }^{8}$ D.J. Lockwood et al., Phys. Rev. Lett. 77, 354 (1996).

${ }^{9}$ T.H. Oosterkamp et al., Phys. Rev. Lett. 82, 2931 (1999).

${ }^{10}$ T. Fujisawa et al., Phys. Rev. Lett. 88, 236802 (2002).

${ }^{11}$ M. Onoda et al., Phys. Rev. B 64, 235315 (2001). 